\title{
Revista da Escola de Enfermagem da Universidade de São Paulo - 36 anos zelando pela qualidade do trabalho na Enfermagem
}

\author{
NURSING SCHOOL OF USP JOURNAL - 36 YEARS LOOKING AFTER QUALITY ON NURSING WORK \\ REVISTA DE LA ESCUELA DE ENFERMERÍA DE LA UNIVERSIDAD DE SÃO PAULO - 36 AÑOS \\ CUIDANDO DE LA CALIDAD DEL TRABAJO EN LA ENFERMERÍA
}

\section{Maria Júlia Paes da Silva1, Margareth Ângelo ${ }^{2,}$ Valéria Castilho ${ }^{3}$, Emiko Yoshikawa Egry ${ }^{4}$, Semiramis Melani Melo Rocha ${ }^{5}$, Piotr Trzeniak ${ }^{6}$}

\author{
RESUMO \\ A REEUSP é uma revista \\ trimestral desde 1998, que \\ publica de 11 a 13 artigos por \\ número nas diferentes subáreas \\ da Enfermagem, na sua maioria \\ sobre ensino, tecnologia em \\ saúde, saúde do adulto (nas \\ suas diferentes especialidades), \\ saúde mental e psiquiátrica. $O$ \\ enfoque dos artigos e pesquisas \\ continua a ser hospitalar. Por \\ estar ligada a uma unidade de \\ ensino, apresenta qualidade nas \\ suas publicações, muitas vezes \\ geradas de teses e dissertações. \\ Enfrenta o desafio de estimular \\ a publicação de autores de \\ outras instituições, para não se \\ tornar endógena. Nos últimos \\ 10 anos recebeu e publicou \\ artigos de 18 estados \\ brasileiros e de várias cidades \\ do interior de São Paulo. Seu \\ projeto gráfico também foi \\ remodelado para 2003, \\ permitindo maior legibilidade e \\ facilidade na leitura. Aceita \\ permuta com outros periódicos \\ e é indexada em várias Bases \\ de Dados. Recebeu a classifi- \\ cação Cinternacional pela \\ CAPES.
}

\begin{abstract}
REEUSP has been a trimestrial journal since 1998, which publishes 11 to 13 articles per issue on different nursing subareas; most of them on teaching, health technology, adult health (in its different specialties), mental and psychiatric health. The article and research emphasis keeps on the hospital. For being linked to a teaching unit, it presents quality on its publishing; many times produced from thesis and dissertations. It faces the challenge of stimulating the publishing of authors from other institutions, for not becoming endogenous. On the last 10 years, it received and published articles from 18 Brazilian states and several country cities from Sao Paulo State. Its graphic design was also remodeled for 2003, allowing higher clearness and easiness for reading. It accepts exchange with other journals and it is indexed on many basis. It was gotten a C international classification by CAPES.
\end{abstract}

\section{KEYWORDS}

Journal.

Publishing.

Scientific Investigation.

\section{RESUMEN}

La REEUSP es una revista trimestral que desde 1998 publica entre 11 a 13 artículos por número en las diferentes sub áreas de la enfermería, en su mayoría sobre enseñanza, tecnología en salud, salud del adulto (en sus diferentes especialidades), salud mentaly psiquiátrica. El enfoque de los articulos e investigaciones continúa siendo hospitalario. Por estar ligada a una unidad de enseñanza, presentan calidad en sus presentaciones, muchas veces generadas de tesis de maestria y de doctorado. Enfrenta el desafio de estimular la publicación de autores de otras instituciones, para que no se torne endógena. En los últimos 10 años recibió y publicó artículos de 18 estados brasileños y de varias ciudades del interior de San Pablo. Su proyecto gráfico también fue remodelado en el 2003, permitiendo mayor legibilidady facilidad en la lectura. Acepta permuta con otros periódicos y está indexada en muchas bases. Recibio la clasificación C internacional dada por la CAPES.

\section{PALABRAS CLAVE}

Publicaciones periódicas., Firma editora.

Enfermería.

\author{
1 Enfermeira. \\ Professora Livre- \\ Docente do Departa- \\ mento de Enferma- \\ gem Médico-Cirúrgica \\ da Escola de Enferma- \\ gem da USP. \\ 2 Enfermeira. \\ Professora Titular do \\ Departamento de \\ Enfermagem \\ Materno-Infantil e \\ Psiquiátrica da \\ EEUSP. \\ angelm@usp.br \\ 3 Enfermeira. \\ Professora Doutora \\ do Departamento de \\ Orientação Profis- \\ sional da EEUSP. \\ valeriac@usp.br \\ 4 Enfermeira. \\ Professora Titular do \\ Departamento de \\ Enfermagem em \\ Saúde Coletiva da \\ EEUSP. \\ emiegry@usp.br \\ 5 Enfermeira. \\ Professora Titular da \\ EERP-USP e \\ visitante na \\ Universidade Federal \\ de São Carlos. \\ smmrocha@eerp.usp.br \\ 6 Físico. Professor \\ Titular da Universi- \\ dade Federal de \\ Itajubá. \\ piotrze@unifei.edu.br
}

Publicações periódicas.

Editoração.

Enfermagem. 
Maria Júlia Paes da Silva Margareth Ângelo

Valéria Castilho

Emiko Yoshikawa Egry

Semiramis Melani M Rocha

Piotr Trzeniak
A comunidade internacional de pesquisadores de Enfermagem e áreas correlatas estão convidadas a publicar na Revista da Escola de Enfermagem da Universidade de São Paulo - REEUSP. Neste periódico, a pesquisa circula com tradição, agilidade, rigor e visibilidade.

A tradição vem dos 36 anos ininterruptos de circulação. A periodicidade, trimestral, e a média de 12 artigos, por fascículo, garantem a agilidade - a pesquisa não fica esperando meses para circular. A retaguarda, propiciada pela Escola de Enfermagem da USP, pelo Conselho Editorial (composto de quatro professores titulares, um livre-docente e um doutor) e pelos pareceristas, $78 \%$ brasileiros e $22 \%$ do exterior, assegura o máximo rigor em termos do conhecimento publicado. As indexações, tanto as já existentes (MEDLINE, LILACS, CINAHL, CUIDEN,
Índice de Revistas Latino-Americanas em Ciências e BDEnf), como as que estão sendo buscadas (incluindo a SciElo), proporcionam visibilidade crescente a cada número.

A Coordenação de Aperfeiçoamento de Pessoal de Nível Superior - CAPES, órgão federal que avalia todo o sistema brasileiro de pós-graduação e, para isso, classifica os periódicos científicos, coloca a REEUSP como Internacional C.

São aceitos para publicação os originais em português, inglês e espanhol. Até hoje a língua portuguesa é predominante, mas os leitores brasileiros têm, em geral, fluência nos três idiomas. Os temas mais abordados pelos autores são os referentes ao ensino de enfermagem, saúde do adulto (nas suas diferentes especialidades) e saúde mental, com ênfase na área hospitalar.

Tabela 1 - Número de artigos por área temática encaminhados à REEUSP, no período de 2000 a 2003

\begin{tabular}{|c|c|c|c|c|c|c|c|c|c|c|}
\hline \multirow{2}{*}{$\begin{array}{c}\text { Áreas } \\
\text { Temáticas }\end{array}$} & \multicolumn{2}{|c|}{2000} & \multicolumn{2}{|c|}{2001} & \multicolumn{2}{|c|}{2002} & \multicolumn{2}{|c|}{ 2003* } & \multicolumn{2}{|c|}{ Total } \\
\hline & $\mathbf{n}^{\mathbf{o}}$ & $\%$ & $\mathbf{n}^{\mathbf{o}}$ & $\%$ & $\mathbf{n}^{\mathbf{o}}$ & $\%$ & $\mathbf{n}^{\mathbf{o}}$ & $\%$ & $\mathbf{n}^{\mathbf{o}}$ & $\%$ \\
\hline Administração & 7 & 13,2 & 10 & 16,9 & 5 & 6,0 & 9 & 13,6 & 31 & 11,9 \\
\hline $\begin{array}{l}\text { Área Básica (Farmaco, } \\
\text { Fisiologia e Biologia }\end{array}$ & 1 & 1,9 & 1 & 1,7 & 0 & 0,0 & 1 & 1,5 & 3 & 1,1 \\
\hline Assistência Domiciliar & 0 & 0,0 & 0 & 0,0 & 5 & 6,0 & 1 & 1,5 & 6 & 2,3 \\
\hline $\begin{array}{l}\text { Centro Cirúrgico e Centro de } \\
\text { Material }\end{array}$ & 0 & 0,0 & 4 & 6,8 & 3 & 3,6 & 3 & 4,5 & 10 & 3,8 \\
\hline Doenças Transmissíveis & 1 & 1,9 & 4 & 6,8 & 0 & 0,0 & 5 & 7,6 & 10 & 3,8 \\
\hline $\begin{array}{l}\text { Ensino(Superior, Nível Médio e } \\
\text { Pós-Graduação) }\end{array}$ & 5 & 9,4 & 9 & 15,3 & 8 & 9,6 & 7 & 10,6 & 29 & 11,1 \\
\hline Ginecologia e Obstetrícia & 5 & 9,4 & 2 & 3,4 & 3 & 3,6 & 6 & 9,1 & 16 & 6,1 \\
\hline Pediatria e Neonatologia & 3 & 5,7 & 3 & 5,1 & 5 & 6,0 & 3 & 4,5 & 14 & 5,4 \\
\hline $\begin{array}{l}\text { Práticas Complementares de } \\
\text { Saúde }\end{array}$ & 1 & 1,9 & 2 & 3,4 & 1 & 1,2 & 3 & 4,5 & 7 & 2,7 \\
\hline Saúde da Mulher & 3 & 5,7 & 4 & 6,8 & 5 & 6,0 & 4 & 6,1 & 16 & 6,1 \\
\hline $\begin{array}{l}\text { Saúde do Adulto (Oncologia, } \\
\text { Hipertensão, Lesões...) }\end{array}$ & 6 & 11,3 & 9 & 15,3 & 16 & 19,3 & 6 & 9,1 & 37 & 14,2 \\
\hline Saúde do Idoso & 3 & 5,7 & 1 & 1,7 & 1 & 1,2 & 3 & 4,5 & 8 & 3,1 \\
\hline $\begin{array}{l}\text { Saúde Mental e Enfermagem } \\
\text { Psiquiátrica }\end{array}$ & 9 & 17,0 & 2 & 3,4 & 15 & 18,1 & 5 & 7,6 & 31 & 11,9 \\
\hline Saúde Pública & 1 & 1,9 & 0 & 0,0 & 3 & 3,6 & 3 & 4,5 & 7 & 2,7 \\
\hline Tecnologia em Saúde & 3 & 5.7 & 2 & 3,4 & 7 & 8,4 & 3 & 4,5 & 15 & 5,7 \\
\hline $\begin{array}{l}\text { Teoria e Pesquisa de } \\
\text { Enfermagem }\end{array}$ & 2 & 3,8 & 2 & 3,4 & 3 & 3,6 & 3 & 4,5 & 10 & 3,8 \\
\hline UTI e Emergência & 3 & 5,7 & 4 & 6,8 & 3 & 3,6 & 1 & 1,5 & 11 & 4,2 \\
\hline Total & 53 & 100 & 59 & 100 & 83 & 100 & 66 & 100 & 261 & 100 \\
\hline
\end{tabular}

* Recebidos até setembro de 2003.

Fonte: arquivos da Secretaria da REEUSP, 2003.

Em sintonia com seu tempo, a REEUSP acabou de passar por uma reformulação estrutural e gráfica e está, em 2003, ainda mais interessante. Desde a diagramação até as capas, que ganharam novas cores, diferentes a cada número do ano - verde, amarela, vermelha e azul - cores que serão mantidas, identificando cada trimestre e facilitando a sua localização no acervo das bibliotecas. 
A Revista aceita diferentes tipos de colaboração, com correspondente diferença na forma, conteúdo e abordagem das matérias, alocadas em seções. Além das seções tradicionais (relatos de pesquisa, estudos teóricos, relatos de experiência profissional e revisões críticas da literatura), foram criadas as de cartas à editora, notas e discussão e, em especial, a que dominamos à beira do leito, que relatará questões com respostas objetivas sobre condutas práticas. Uma breve descrição de cada uma dessas seções será dada a seguir:

- Relatos de pesquisa: investigações baseadas em dados empíricos, utilizando metodologia científica. Limitados a 15 páginas impressas em formato final, incluindo resumo, abstract, figuras, tabelas e referências.

- Estudos teóricos: análise de construtos teóricos, levando ao questionamento de modelos existentes e à elaboração de hipóteses para futuras pesquisas. Especificações gerais idênticas às dos Relatos de pesquisa.

- Relatos de experiência profissional: estudos de caso, contendo análise de implicações conceituais, ou descrição de procedimentos com estratégias de intervenção, evidência metodológica apropriada de avaliação de eficácia, de interesse para a atuação de enfermeiros em diferentes áreas. Especificações gerais idênticas às dos Relatos de pesquisa, mas limitados a 10 páginas.

- Revisões críticas da literatura: análise abrangente de literatura sobre um assunto de interesse para o desenvolvimento da Enfermagem. Especificações gerais idênticas às dos Relatos de pesquisa, mas limitados a 10 páginas.

- Notas e Discussão: colaborações de caráter geral ou de discussão de artigos publicados, necessariamente com enfoque científico (não opinativos). Limitadas a 2 páginas impressas, sem resumo e sem abstract.

- Resenhas: revisões críticas de obra recém-publicada ou equipamentos e materiais recém-lançados, orientando o leitor quanto as suas características, limitações e emprego potencial. Limitadas a 2 páginas impressas em formato final.

- À beira do leito: questões com respostas objetivas sobre condutas práticas.
- Cartas à Editora: contêm sugestões, comentários sobre trabalhos publicados, opiniões, dúvidas, perguntas, com o propósito de contar com a participação do leitor. No máximo meia página.

- Erratas: após a publicação do artigo, se as autoras e autores identificarem a necessidade de uma errata, deverão enviá-la à Secretaria da REEUSP imediatamente, de preferência por e-mail.

Ao longo dos seus 36 anos, a Revista foi alvo de análises críticas e avaliações por parte de suas editoras, as Diretoras da Escola de Enfermagem da USP, ainda que de maneira assistemática. Na primeira avaliação, datada de 1982, foi feito um levantamento sobre a utilização dos periódicos na Biblioteca da Escola de Enfermagem da USP (a maior da América Latina na área). Constatou-se que, de janeiro a dezembro de 1981, a REEUSP foi o segundo periódico mais procurado naquela biblioteca, com um total de 707 consultas $^{(1)}$.

O resultado de uma avaliação informal, abrangendo o período de 1982 a 1986, foi publicado sob forma de editorial no v. 21, n.1, de 1987. Nele, a Diretoria da Escola de Enfermagem da época descreveu as finalidades e as características da Revista e ressaltou que, no início, seu conteúdo foi mais voltado para assistência e ensino, tendo-se enfocado progressivamente para a investigação científica, durante a década de $70^{(2)}$.

No final de 1987, houve outra publicação dos integrantes do Conselho Editorial da Revista, analisando os números publicados. Foi verificado que ${ }^{(3)}$ :

- o enfoque dos artigos e pesquisas continuou a ser da área hospitalar;

- houve aumento no número de pesquisas da áreas de ensino e administração;

- a maior parte dos autores foi de enfermeiros com o título de mestre.

O último ponto não é de se estranhar, uma vez que, além dos enfermeiros de campo escreverem menos que os docentes, naquela época havia poucos mestres no campo - eram mais da docência, e o número de cursos de Mestrado era maior que o de Doutorado - como ainda é hoje.

A quantidade de artigos de autoria dos pesquisadores da Escola de Enfermagem da
Revista da Escola de

Enfermagem da

Universidade de

São Paulo - 36 anos

zelando pela

qualidade do trabalho

na enfermagem 
Maria Júlia Paes da Silva Margareth Ângelo

Valéria Castilho

Emiko Yoshikawa Egry

Semiramis Melani M Rocha

Piotr Trzeniak
USP tem sido uma crítica e um alerta constante. No entanto, pode-se verificar, pela Tabela 2, uma participação considerável e

Tabela 2 - Distribuição do número dos autores que publicaram nos últimos 5 anos na REEUSP, de acordo com a instituição de origem

\begin{tabular}{|c|c|c|c|c|c|c|c|c|c|c|}
\hline \multirow{2}{*}{$\begin{array}{c}\text { Ano } \\
\text { Autores }\end{array}$} & \multicolumn{2}{|c|}{1999} & \multicolumn{2}{|c|}{2000} & \multicolumn{2}{|c|}{2001} & \multicolumn{2}{|c|}{2002} & \multicolumn{2}{|c|}{$2003^{*}$} \\
\hline & $\mathbf{n}^{\mathbf{o}}$ & $\%$ & $\mathbf{n}^{\mathbf{o}}$ & $\%$ & $\mathbf{n}^{\mathbf{o}}$ & $\%$ & $\mathbf{n}^{\mathbf{o}}$ & $\%$ & $\mathbf{n}^{\mathbf{o}}$ & $\%$ \\
\hline $\begin{array}{l}\text { Da Escola de } \\
\text { Enfermagem da USP }\end{array}$ & 43 & 38,1 & 52 & 39,1 & 54 & 36,0 & 53 & 41,7 & 19 & 31,6 \\
\hline $\begin{array}{l}\text { De outras instituições de } \\
\text { ensino, pesquisa e } \\
\text { assistência do Brasil }\end{array}$ & 70 & 61,9 & 81 & 60,9 & 96 & 64,0 & 73 & 57,5 & 40 & 66,7 \\
\hline $\begin{array}{l}\text { De instituições de ensino, } \\
\text { pesquisa e } \\
\text { assistência do exterior }\end{array}$ & 0 & 0 & 0 & 0 & 0 & 0 & 1 & 0,8 & 1 & 1,7 \\
\hline Total & 113 & 100 & 133 & 100 & 150 & 100 & 127 & 100 & 60 & 100 \\
\hline
\end{tabular}

* Recebidos até setembro de 2003

Fonte: Arquivos da Secretaria da REEUSP, 2003.
Podemos perceber que a maioria dos artigos publicados não é de docentes da Escola de Enfermagem da USP, demonstrando, assim, a tentativa da Revista não ser endógena, além de ser valorizada pelos enfermeiros e docentes de outras instituições.

Na Tabela 3, observamos a distribuição dos autores brasileiros que encaminharam crescente de autores de outras instituições de ensino, nos últimos 5 anos.
Tabela 3 - Distribuição dos autores nacionais que encaminharam artigos à REEUSP no período de 1998 a 2003, segundo a procedência por regiões do Brasil

\begin{tabular}{lcc}
\hline Regiões do Brasil & $\mathbf{n}^{\mathbf{o}}$ & $\mathbf{\%}$ \\
\hline Região Norte & 4 & 0,38 \\
Região Centro-Oeste & 25 & 2,38 \\
Região Nordeste & 38 & 3,60 \\
Região Sudeste & 962 & 91,45 \\
Região Sul & 23 & 2,19 \\
\hline Total & $\mathbf{1 0 5 2}$ & $\mathbf{1 0 0 \%}$ \\
\hline
\end{tabular}


Outro dado interessante é o total de trabalhos publicados nos últimos 5 anos, originados Outro dado interessante é o total de trabalhos publicados nos últimos 5 anos originados de de teses ou dissertações: 11 (22,9\%) em 1999; 11 (20\%) em 2000; 5 (8,6\%) em $2001 ; 23(44,2 \%)$ em 2002 e $8(33,3 \%)$ em 2003. Podemos afirmar que os cursos de pósgraduação em Enfermagem, atualmente com 30 anos de existência no Brasil, contribuem de forma significativa para o aumento da produção científica em Enfermagem.

A fonte de recursos para a edição das revistas tem sido o Programa de Apoio às Publicações Científicas da USP (SIBI) e os assinantes, que cobrem as despesas de 3 dos 4 números anuais. Houve 290 assinaturas em 2000; 306 em 2001; 331 em 2002 e 306 em 2003.

As dificuldades encontradas para a publicação da REEUSP são:

- a divulgação do conhecimento em português - idioma pouco conhecido no exterior, dificultando a difusão dos nossos trabalhos em outros países (pois, o inglês é a língua dominante na comunidade científica em Enfermagem);

- custo - ainda não somos totalmente auto-suficientes, dependemos de auxílio externo para publicação;
- a regionalização dos autores - os programas de pós-graduação estão concentrados na Região Sudeste.

Ressaltamos, entretanto, que não só o progresso da ciência como o da tecnologia estão intrinsecamente articulados às políticas do desenvolvimento e ciência do País. A pós-graduação é fruto de uma necessidade do estado de crescimento de toda uma cadeia de produção: indústrias, serviços, cultura... que clama por novos conhecimentos. As pesquisas nascem das necessidades de resolver problemas e cada região, de acordo com suas próprias características e recursos, está construindo seu modo de pesquisar. Não é só a universidade que produz pesquisa: outros setores da sociedade também o fazem, inclusive o setor de segurança nacional.

Essa análise sintética nos mostra que a REEUSP está crescendo e tem o reconhecimento da comunidade científica. Ela se constitui, portanto, num veículo adequado para publicar os resultados da pesquisa em Enfermagem, em todas as suas subáreas, originárias de qualquer país e atende a leitores diversificados, comprometidos com o avanço do conhecimento!

\section{REFERÊNCIAS}

(1) Aguiari CSAL, Hondo MMV. A utilização dos periódicos na biblioteca da Escola de Enfermagem da USP. Rev Esc Enferm USP 1982; 16(1):5-16.

(2) Pinheiro MRS. Vinte anos de vida [editorial] Rev Esc Enferm USP 1987; 21(1):1-2.
Revista da Escola de

Enfermagem da

Universidade de

São Paulo - 36 anos

zelando pela

qualidade do trabalho

na enfermagem
(3) Margarido AS; Koizumi MS; Rolim AM; Fukuda IMK. Análise da Revista da Escola de Enfermagem da USP: período de 1982 a 1986. Rev Esc Enferm USP 1987; 21(3):285-91. 